\title{
Semi-automated Basal Ganglia Segmentation Using Large Deformation Diffeomorphic Metric Mapping
}

\author{
Ali Khan ${ }^{1}$, Elizabeth Aylward ${ }^{2}$, Patrick Barta ${ }^{3}$, \\ Michael Miller ${ }^{4}$, and M. Faisal Beg ${ }^{1}$ \\ 1 School of Engineering Science, Simon Fraser University, \\ 8888 University Drive, Burnaby BC, V5A 1S6, Canada \\ 2 Department of Radiology and Psychiatry, \\ University of Washington, Box 357115, Seattle, WA 98195, USA \\ 3 Department of Psychiatry and Behavioral Sciences, \\ Division of Psychiatric Neuro-Imaging, \\ The Johns Hopkins University School of Medicine, Baltimore, MD 21287, USA \\ 4 Center for Imaging Science, \\ The Johns Hopkins University, 301 Clark Hall, Baltimore, MD 21218, USA
}

\begin{abstract}
This paper investigates the techniques required to produce accurate and reliable segmentations via grayscale image matching. Finding a large deformation, dense, non-rigid transformation from a template image to a target image allows us to map a template segmentation to the target image space, and therefore compute the target image segmentation and labeling. We outline a semi-automated procedure involving landmark and image intensity-based matching via the large deformation diffeomorphic mapping metric (LDDMM) algorithm. Our method is applied specifically to the segmentation of the caudate nucleus in pre- and post-symptomatic Huntington's Disease (HD) patients. Our accuracy is compared against gold-standard manual segmentations and various automated segmentation tools through the use of several error metrics.
\end{abstract}

\section{Introduction}

Shape and volume change of the basal ganglia structures, which include the caudate nucleus and putamen, have been the focus of investigation in clinical studies of Huntington's Disease [1] and other neuro-degenerative disorders. In order to study shape and volume changes, segmentation of the basal ganglia structures in an MRI image is the first processing step. Manual segmentation by a trained rater is the current anatomic gold standard, but this technique requires a sizable amount of time from an anatomic expert. Manual segmentations by different anatomic experts also suffer from inter-rater reliability issues. Reliable and accurate semi-automated segmentation of the caudate nucleus is limited by the following factors: limited resolution of MRI scans, inhomogeneous intensities throughout the caudate, and ill-defined boundaries. Successful segmentation algorithms built on automatic to semi-automatic algorithms making use of some form of expert prior knowledge are likely to overcome the mentioned difficulties. 
The LDDMM tool 2] provides non-rigid registration between two grayscale anatomical images, which we use to develop our system for automated segmentation. Given a template image, target image, and template segmentation, the non-rigid mapping of the template image to the target image can be used to propagate the template segmentation, generating a target segmentation. In this way, we are able to use anatomic expert priors, namely a manually generated template segmentation, to produce target segmentations. In this paper, we present the validation of this method for accuracy and reliability.

\section{Method}

\subsection{Data}

MRI scans used for this study were all acquired on a GE 1.5T scanner. Three types of scans were analyzed: (1) $1.5 \mathrm{~mm}$ SPGR (spoiled gradient recalled echo in steady state) coronal series, $\mathrm{TR}=35 ; \mathrm{TE}=5, \mathrm{NEX}=1$, flip angle $=45^{\circ}$, voxel size $=0.9735 \mathrm{~mm}$ in $\mathrm{x}$ and $\mathrm{y}$ direction, from subjects with known caudate atrophy (patients with Huntington's disease); (2) $1.5 \mathrm{~mm}$ SPGR axial series, TR $=18 \mathrm{TE}=3, \mathrm{NEX}=2$, field of view $=24 \mathrm{~cm}$, flip angle $=20^{\circ}$, voxel size $=$ $0.9735 \mathrm{~mm}$ in $\mathrm{x}$ and $\mathrm{y}$ direction, from subjects with possible caudate atrophy (pre-symtomatic carriers of the HD gene); and (3) $1.2 \mathrm{~mm}$ SPGR sagittal series, $\mathrm{TR}=11.1, \mathrm{TE}=2.2, \mathrm{NEX}=1$, field of view $=24 \mathrm{~cm}$, flip angle $=25^{\circ}$, voxel size $=0.9735 \mathrm{~mm}$ in $\mathrm{x}$ and $\mathrm{y}$ direction, from children with no known caudate atrophy.

Scans were of varying levels of quality for the segmentation program (with the $\mathrm{NEX}=2$ scans have the most defined borders between caudate and white matter) and represented groups will have varying levels of caudate volume. Scans were stripped of all identifying information.

Computation of automated segmentations via image matching involves the use of a template and a target image. Our Series 1 data consisted of patients scanned on two separate occasions, therefore one scan was chosen as a template image, and the other scan was chosen as a target image. Series 2 and Series 3 data did not include multiple scans from the participants, restricting us from using the same patient as template and target. For the Series 2 data, one patient image was chosen as a template to compute segmentations for the other Series 2 patient images. Series 3 segmentations were computed similarly. In total, 17 automated segmentations were produced, five Series 1, seven Series 2, and five Series 3.

\subsection{Registration and Pre-processing}

Global alignment of the basal ganglia structures is required before intensitybased image matching can take place; rigid landmark-based registration was used to accomplish this task. We chose to place landmarks on the surfaces of the lateral ventricles because of their unique properties: adjacency to the caudate nucleus during all stages of atrophy, well-defined boundaries, and homogeneous intensities within these boundaries. Furthermore, placement of landmarks on a 
3D surface as opposed to a 3D volume limits the degrees of freedom in placement hence resulting in lower variability. Aside from registration, landmarks placed on the ventricular surface are subsequently used in image matching to calculate an initial template to target mapping which is later refined by image intensity-based alignment. The ventricle segmentation used for surface generation is produced by global thresholding with user guidance and takes a trained user approximately five minutes to complete.

Landmarks are placed interactively by the user on the inferior surface of the ventricles, which is adjacent to the superior surface of the caudate nucleus. Landmarks are placed on the ventricular surface of the template first, then the target; the user attempting to place the landmarks in the same relative location on the surface. After placing 6-7 landmarks on each side of the ventricles, the target landmarks on the left target ventricle are rigidly deformed to the template landmarks on the left template ventricle, generating the target image deformed in accordance with the left set of landmarks. The landmarks on the right ventricles are transformed in the same manner producing another target image deformed in accordance with the right set of landmarks. A six-parameter translation/rotation least squares landmark matching algorithm is used to rigidly rotate and translate the landmarks and the corresponding images.

After the left and right rigid transformations, the left template and target caudates, and the right template and target caudates are globally aligned. We then define a region of interest (ROI) to include the caudate nuclei. We isolate our ROI by inspecting the boundaries of the caudates in the template image and cropping a bounding box surrounding these areas. The template and target images are then preprocessed with edge-preserved smoothing [3], histogram matching, and intensity stretching for noise reduction, intensity matching and contrast enhancement respectively.

\subsection{Image Matching}

Image matching is achieved through the solution of the large deformations diffeomorphic metric mapping (LDDMM); a detailed derivation and discussion of this can be seen in [2]. Template and target images, $I_{0}$ and $I_{1}$, represented by functions $I: \Omega \rightarrow \mathbb{R}$, are mapped via the transformation $\varphi: \Omega \rightarrow \Omega$, where $\Omega \subseteq \mathbb{R}^{3}$. The diffeomorphic transformation generated is smooth and has a smooth inverse, hence, smoothness of anatomical features is preserved and coordinates are transformed consistently.

Globally registered and image intensity equalized images are input into the image matching (LDDMM) program. Our computations were carried out on a 64bit symmetric multi-processor, with computation times not exceeding one hour. Composition of the target segmentation is achieved by composing the template binary segmentation with the appropriate LDDMM mapping. Rigidly deforming the target segmentation back to the patient space and combining the left and right sides produces the final automated segmentation.

A graphical summary of our method for obtaining a mapping between a template and target image is shown in Figure 1. 


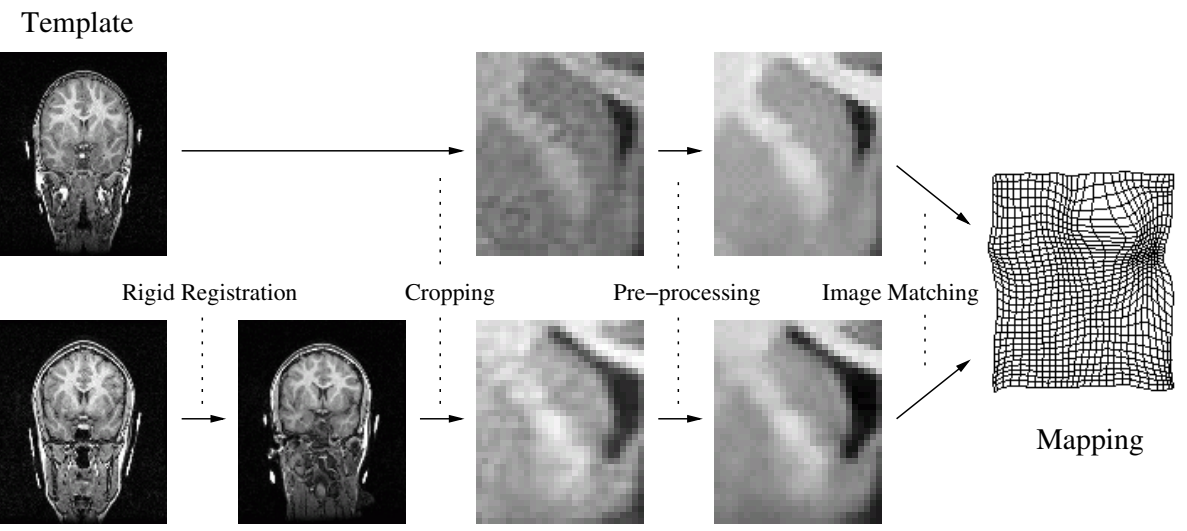

Target

Fig. 1. Procedural flow outlining the generation of a template to target diffeomorphic mapping for one side (left) of the brain. The target is deformed rigidly to the template, both images are cropped and pre-processed, and LDDMM image matching is used to compute the final mapping. Images shown are representative slices taken from a Series 3 patient.

\section{Results}

To facilitate comparison of our continuous automated segmentations with the binary manual segmentations, the manual segmentations are slightly smoothed. The L1-distance between segmentations is reported as the L1 error, and caudate volumes are also computed.

We have also developed a boundary distance metric which aims to provide correspondence between two surfaces by computing the LDDMM mapping from an automated segmentation to a manual segmentation. Applying this mapping to the template surface displaces each point on the template to its corresponding point on the target surface. Since the LDDMM matching is in the inexact framework, a template surface point may not map exactly to a target surface point. However since the deformed template surface closely approximates the target surface, choosing the nearest point on the target surface to a point on the template surface is likely to provide very good correspondence. Using this correspondence we can compute the boundary distances for each point on the template surface to a point on the target surface. The average for the surface is calculated and is reported as the mean boundary distance.

Another estimate of surface distance, namely the maximum surface distance, is given by the Hausdorff distance. Calculation of this metric is carried out as described in 4 .

Segmentation accuracy results were compiled by computing the metrics described above for each automated segmentation. We report the average and standard deviation of these metrics for each dataset series. These results are shown in Table 1. L1 error and volume error results for individual Series 1 pa- 
Table 1. Caudate nucleus LDDMM segmentation results

\begin{tabular}{|c|c|c|c|}
\hline Error Metric & Series 1 & Series 2 & Series 3 \\
\hline Volume Error (\%) & $5.10 \pm 3.60$ & $8.08 \pm 3.72$ & $7.72 \pm 6.18$ \\
L1 Error (\%) & $27.94 \pm 6.51$ & $36.21 \pm 5.51$ & $30.17 \pm 6.99$ \\
95\% Sym. Hausdorff (mm) & $2.10 \pm 0.98$ & $2.27 \pm 0.75$ & $2.19 \pm 0.70$ \\
Mean Boundary Dist. (mm) & $1.12 \pm 0.57$ & $1.17 \pm 0.47$ & $0.94 \pm 0.20$ \\
\hline
\end{tabular}

This table shows the computed results for our automated segmentations, reporting the mean and standard deviation $(\mu \pm \sigma)$ for each dataset. High L1 error is mainly due to discrepancies on the boundaries of the automated segmentations. Small boundary distance metrics (Hausdorff, mean boundary distance) indicate that the overall shape is accurate
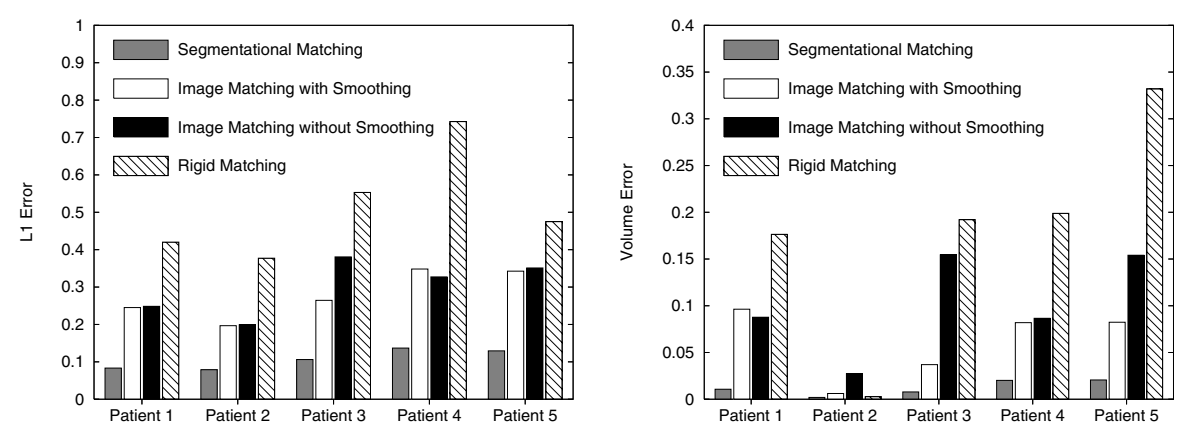

Fig. 2. L1 errors (left) and volume errors (right) for Series 1 segmentations using different transformations. Segmentational matching refers to using binary segmentations as template and target images, image matching with smoothing is our regular system, image matching without smoothing skips the noise-reduction step, and rigid matching does not use any image matching transformation.

tients are shown in Figure 2. We compare results we get choosing from different mappings strategies: rigid rotation/translation, intensity-based image matching with and without edge-preserving smoothing, and pure segmentational matching. Representative slices for two different Series 1 patients displaying automated and manual caudate segmentations are shown in Figure 3.

Our caudate nucleus segmentation results are in the same range as other comparable methods reported in literature [5], with less requirements on parameter tuning and user input.

Proven reliability of our segmentation system is essential for its use in clinical studies. Our reliability study consisted of performing the segmentation procedure 8 times each for two different Series 2 patients, generating a new caudate nucleus segmentation each time. Ideally, for an inter-rater reliability calculation, 8 different raters would rate each patient, however, we were limited to using a single rater, computing segmentations on different days. For each image matching computation (total of 16), the ventricles were segmented, landmarked, and the 


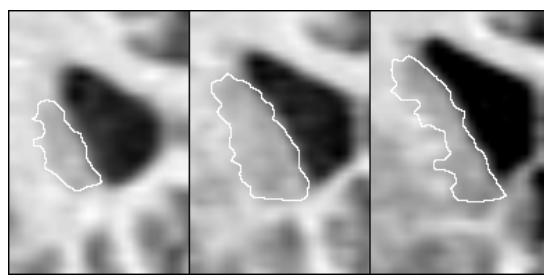

Patient A - automated segmentation

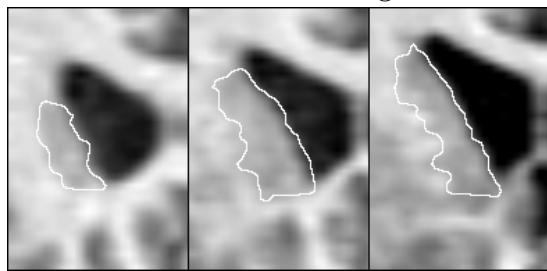

Patient A - manual segmentation

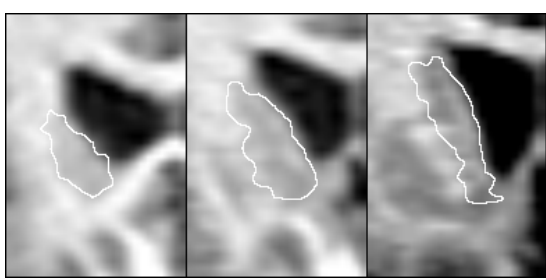

Patient B - automated segmentation

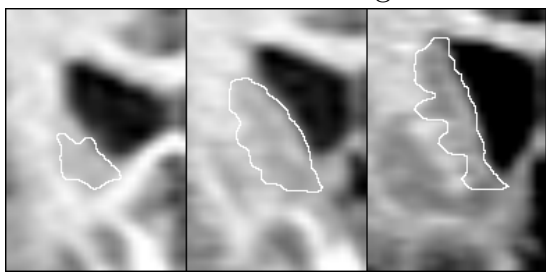

Patient B - manual segmentation

Fig. 3. Three coronal slices of the left caudate nucleus of two Series 1 patients shown with automated binary threshold segmentations (Top) and manual segmentations (Bottom) outlined in white. (Left) Patient A: L1 error $=26.45 \%$, mean boundary distance $=0.6223 \mathrm{~mm}$. (Right) Patient B: L1 error $=34.82 \%$, mean boundary distance $=$ $1.3409 \mathrm{~mm}$.

average caudate nucleus intensity was chosen; as usual, the same template image was used for all segmentations. Segmentations for the left and right caudates were derived for each of these cases, and their volumes were used to calculate the intra-class correlation coefficient. Although technically an intra-rater reliability coefficient, because each segmentation was done on a different day, it is our best approximation to inter-rater reliability. This is because the day to day variability for a single rater segmenting the ventricles, landmarking and choosing the average caudate intensity should be similar to the variability between different raters, especially when the system as a whole is considered.

The simple replication reliability study uses a one-way random effects model to calculate the intra-class correlation coefficients (ICC) and confidence intervals for the caudate nucleus volumes as dictated by [6]. The calculated ICC for Series 2 caudates is 0.937 with a $95 \%$ confidence interval of $(0.759,0.994)$. According to [7], ICC $>0.74$ corresponds to excellent reliability, which suggests our segmentation system is sufficiently reliable.

\section{Discussion}

One difficulty in producing automated segmentations of the caudate nucleus is determining the location of the most inferior axial slice. This is defined to be the most inferior slice when the caudate and putamen are visibly separated by the internal capsule with the brain aligned in AC-PC orientation. Generating an automated segmentation which follows the same strict guidelines is difficult at 
best, and determining the inferior axial cut-off is a task better suited for the user. Using a template segmentation which includes the caudate nucleus, putamen, and the nucleus accumbens would produce a target segmentation that can be manually bisected to produce caudate and putamen segmentations cut-off at the correct axial plane.

User involvement in our semi-automated segmentation scheme is minimal and does not require expertise in brain anatomy. The user is necessary for four tasks: 1. automated thresholding-based segmentation of the ventricles, 2. landmarking of the ventricular surfaces, 3. definition of the bounding box to delineate the image region for matching, and 4. identification of average caudate intensities for intensity equalization. As discussed earlier, our procedure for lateral ventricle segmentation is fast and easy since high accuracy is unnecessary as only a small area of the surface is used for landmarking. Placing landmarks is also an easy task once landmark positions have been standardized. Definition of a bounding box is only necessary for template images, as image registration should ensure that the target images will fit in the template bounding box by always padding the template bounding box by a tolerance of about 10 voxels or such. Identifying the average intensity for the target structures is subject to some variability, but as our reliability study shows, these variations do not amount to notable changes in the segmentations.

The automated and the manual segmentations are found to differ on the exterior boundary of the caudate. Due to the elongated, narrow shape of the caudate nucleus, the ratio of the number of voxels on the surface of the caudate to the total number of caudate voxels is very high, on average being $71 \pm 9 \%$. Therefore, the partial volume effects are likely to be a heavy influence in calculation of the L1 distance which measures the difference in voxel labeling between the automated and the manual segmentation, especially since there are a large number of these on the exterior of the caudate that are not exactly matched due to regularization constraints placed in computing dense diffeomorphic transformations. Examination of Figure 3 reveals that accurate delineation of the caudate nuclei is still present in segmentations possessing relatively high L1-distance errors. Further improvement in accuracy is likely through the use of a template image representing the average of the study population. We are currently working on geodesic shooting methods in LDDMM to produce an average image.

Concluding, the results we have shown demonstrate that our semi-automated image matching system reliably segments the caudate nucleus with accuracy comparable to current automated methods. Segmentation of the basal ganglia via image matching is the initial step in shape analysis as the diffeomorphic mapping defines the necessary correspondence between images. Application of our procedure to other structures should also prove successful, as our findings indicate that the general image matching segmentation concept is robust.

\section{Acknowledgments}

We acknowledge funding support from NSERC 31-611387 for this work. 


\section{References}

1. Aylward, E., Rosenblatt, A., Field, K., Yallapragada, V., Kieburtz, K., McDermott, M., Raymond, L., Almqvist, E., Hayden, M., Ross, C.: Caudate volume as an outcome measure in clinical trials for huntington's disease: a pilot study. Brain Research Bulletin 62 (2003) 137-141

2. Beg, M.F., Miller, M.I., Trouvé, A., Younes, L.: Computing large deformation metric mappings via geodesic flows of diffeomorphisms. IJCV 61 (2005) 139-157

3. Whitaker, R., Xue, X.: Variable-conductance, level-set curvature for image denoising. (2001)

4. Gerig, G., Jomier, M., Chakos, M.: Valmet: A new validation tool for assessing and improving 3D object segmentation. (2001)

5. Pitiot, A., Delingette, H., Thompson, P., Ayache, N.: Expert knowledge guided segmentation system for brain MRI. NeuroImage 23 (2004) S85-S96 Special Issue: Mathematics in Brain Imaging.

6. Fleiss, J.L.: The Design and Analysis of Clinical Experiments. Wiley-Interscience (1999)

7. Fleiss, J.L.: Statistical Methods for Rates and Proportions. Wiley (1981) 